\title{
The new genetics in psychiatry Eleanor Feldman
}

Advances in the understanding of the genetic causes of some neuropsychiatric disorders are having an impact on clinical practice as direct mutation analysis becomes possible. Mutation analysis is now available in UK Health Service diagnostic laboratories for Huntington's disease (HD) and the fragile $X$ syndrome (FRAXA). Psychiatrists need to be familiar with issues surrounding presymptomatic and diagnostic testing in $\mathrm{HD}$ and diagnostic and carrier testing in FRAXA. They may be asked to assist clinical geneticists in the assessment of candidates for HD presymptomatic testing and a suggested mode of assessment is described here. The procedure for HD will provide the model for use with other familial neuropsychiatric disorders of late onset, notably familial Alzheimer's disease (FAD). Testing for FAD is already possible in conjunction with research laboratories in the few families where a mutation has been discovered and we shall have more tests for FAD within a few years.

\section{Huntington's disease}

Huntington's disease is an autosomal dominant disorder characterised by bradykinesia, abnormal involuntary movements and progressive dementia particularly involving memory and frontal lobe functions. The basal ganglia show characteristic degeneration (Hayden, 1981). The disorder affects 1 in 10000 individuals in most populations of European origin. Although normally considered a disorder with onset in middle to late years, the severest forms of the illness have been seen in children as young as two years with juvenile onset associated with a preponderance of paternal transmission of the disease gene. First degree relatives of patients with $\mathrm{HD}$ are at $50 \%$ risk, but this risk will generally diminish as a relative ages beyond the age of onset in the proband.

Many patients experience affective disorder, usually depression, but occasionally mania-like episodes, as part of the condition (Folstein et al,
1983); non-affective psychoses also occur (Caine \& Shoulson, 1983; Pflanz et al, 1991). Mental changes such as difficulty in recall, affective disorder, irritability, and poor judgement may precede the appearance of motor signs by some years and diagnosis is often difficult in the early stages of the disorder, especially if a definite family history is not forthcoming (Folstein et al, 1986; Diamond et al, 1992). Psychiatric syndromes should be treated as for functional disorders; in particular, the depressive illnesses may respond to conventional antidepressant treatments (Ford, 1986).

\section{The Huntington's disease gene}

The mutation in HD was described in 1993 after a ten-year gene search utilising positional cloning strategies (Huntington's Disease Collaborative Research Group, 1993). The mutation is in a gene named Huntingtin by its discoverers and lies at the tip of the short arm of chromosome 4 (4p16.3). Huntingtin is widely expressed throughout the body, but its function is unknown. The mutation takes the form of an expanded trinucleotide repeat of the sequence (CAG) where $n$ is any number over 30. Repeats of 30-40 may be found in both nonaffecteds and affecteds.

Repeat size is not a reliable predictor of age of onset as some early-onset cases have been reported with repeats in the 35-50 range; hence, repeat number is not used in genetic counselling (Duyao et al, 1993). Expanded CAG repeats in HD inherited through the male germline are more likely to undergo significant expansion than those inherited through the female germline, explaining the phenomenon of anticipation, i.e. the disorder may have an earlier age of onset in offspring than in the affected parent and, in the case of HD, anticipation occurs when the disorder is inherited through the male line (Duyao et al, 1993).

\section{Presymptomatic testing}

Presymptomatic testing of those at genetic risk of the illness became a reality in 1987 following the discovery of genetic markers tightly linked to the

Dr Eleanor Feldman is a Senior Lecturer in Psychiatry at the University of Nottingham where she provides a neuropsychiatric genetic counselling service in conjunction with the Interdisciplinary Centre for Medical Genetics and a liaison psychiatry service to the Queen's Medical Centre. Prior to this post she held a Wellcome Training Fellowship at the University of Cambridge, researching in laboratory molecular medicine. 
disease gene (Gusella et al, 1983). From the outset, there has been concern about the ethics of presymptomatic testing, given the possible impact on an individual and their spouse of discovering that they had a risk in excess of $95 \%$ of developing an incurable fatal dementing illness. There has been particular concern that such information could result in loss of hope and might precipitate suicide, already known to be a risk in those affected (Schoenfeld et al, 1984). In the US, Canada and the UK, extensive counselling programmes were developed including the administration of psychological and social rating instruments, and, in some centres, full psychiatric assessment. The purpose of the process has been to allow people time and the opportunity to reflect upon the implications of a 'good' or a 'bad' test result and the implications for other family members before they go ahead with testing. It is considered that a detailed counselling protocol is essential for presymptomatic testing (Tyler et al, 1992).

The protocol for predictive testing is described by Craufurd \& Tyler (1992). Contraindications include those with a declared intention or significant risk of suicide or current mental illness if it is likely to be exacerbated by an unfavourable test result. A history of alcohol or drug misuse suggests proceeding with caution and, at least, making special follow-up arrangements. A previous history of mental illness, if recent, may also be an indication to postpone testing. Given these contraindications, specialist psychiatric assessment is advisable as part of the procedure.

\section{Psychiatric consultation in Huntington's presymptomatic counselling}

There are no strict rules about how to approach a candidate coming for psychiatric assessment as part of the presymptomatic counselling protocol. Sometimes siblings ask to be seen together, but I always see each member of a family at risk on their own. The following steps are helpful in practice.

(a) Explore the candidate's understanding of, and feelings about, psychiatric assessment. Candidates come with fantasies and misconceptions, both positive and negative, about the psychiatrist's role.

(b) Correct misconceptions and explain role. Candidates should be assured that this is a routine assessment - emphasise that the main purpose is to assist candidates in exploring the likely psychological impact of the test result and to aid the clinical genetics team in supporting candidates throughout the process. The final decision on testing is a decision reached in agreement with the candidate and the other members of the clinical
Box 1. Clinical features of Huntington's disease

Mean age of onset is 49 years

Mean duration of disease is $\mathbf{1 5}$ years

Motor abnormalities: Chorea and/or rigidity (can be confused with Parkinson's disease)

Mental abnormalities: Personality change, memory impairment and mood disorders

Juvenile form of Huntington's disease:

Develops before age 20

Presentation usually with behaviour disorder or frank psychosis

Rigidity commoner than chorea

Epilepsy in $1 / 3$ to $1 / 2$ cases

Affected parent is usually the father

Disease lasts 5-10 years before death

Cerebellar signs

team involved. Candidates in whom it is considered that the test would be harmful can be helped to be made aware that it is not currently in their best interests to have the test, and the psychiatric assessment provides them with an opportunity to have professional help which they otherwise may not get. The issue of testing is reviewed when their mental state has improved.

(c) Enquire about the illness in the family and family issues. Ask who is affected, what the course of the illness has been, and what problems the family have had in caring for the affected individual(s). If the candidate has too little knowledge of the condition, I often advise them to make contact with the Huntington's Disease Association (address at end of article) and suggest further discussion with the clinical geneticist and/or neurologist involved. The psychiatrist should be careful about giving an oversimplified, and perhaps frighteningly gloomy, picture of the condition as there are a variety of presentations and degrees of severity. In taking the rest of the family history, issues of importance include who else in the family is interested in testing, with whom the disease can or cannot be openly discussed, who is most supportive to the candidate, and the attitude of the candidate's partner or spouse.

(d) Explore motivations for testing, expectations from a result either way, and the anticipated result. Candidates should be asked what effect a test result either way will have on their lives. Many say they prefer certainty to uncertainty, that they will make positive use of the knowledge by not postponing things in life, and will make financial plans for the future. Those with children often say they want to 
know for their children's sake; some without children say they may decide not to have any if they test positive, while others will decide to have children earlier in life. It is important to explore the anticipated outcome as some candidates may have convinced themselves that they will be affected or not affected and planned their lives around a particular future scenario; such individuals will have more difficulty adjusting if the test contradicts their expectation.

(e) Undertake a routine systematic enquiry including personal history and general medical history. This should cover general symptoms of recent worry and tension and the reaction to learning about the family illness. Enquire about panic attacks and anxiety states, obsessional neurosis, depression, mania, alcohol and drug misuse, and memory problems, including simple tests of registration and recall. It is worth paying particular attention to coping strategies, and essential to cover any past or present suicidal ideation or acts of self-harm, current life stresses and the level of support from others.

(f) Share and discuss your advice. Summarise with candidates what you have learned about their coping style and encourage them to think through what they might expect to feel from a test result either way. It should be emphasised that the decision to have a test is theirs and they should not allow themselves to be pressured into a test they do not really want or feel ready for. Sometimes children who are at $25 \%$ risk may pressurise a parent (at $50 \%$ risk) into entering the protocol when he or she may not otherwise wish to know. Many parents will put their children's interests before their own and agree to participate. These parents, who are closer to the age of onset than their children who are driving the process forward, will need particular support. Others, where the family is more dysfunctional, may not be in contact with their children and may thereby be protected from the information they do not wish to hear. These situations can pose uncomfortable ethical dilemmas for the professionals concerned.

(h) Allow a joint session with partner/spouse. Partners may sometimes be more upset by the process and result than candidates at risk of the illness; it is they who bear the brunt of caring for an ill spouse and may have to watch their children deteriorate and die of the same disorder.

The psychiatrist should not tell candidates of suspicions that they are already affected with HD, as personality and affective abnormalities alone have poor predictive value (many children of $\mathrm{HD}$ parents grow up in unhappy or very dysfunctional families); neurological examination is more reliable. Some centres are able to include neuropsychological assessment in the presymptomatic assessments. A definite decline in memory function may be considered diagnostic, however, full neuropsychological assessment is time consuming, requires considerable expertise, and may not alter the management of a particular case; it is not included as part of the UK presymptomatic counselling protocol.

\section{Reactions to the test result}

To date, experience has been that the majority of people have a normal adjustment reaction in response to an increased risk result and do not require specialist psychiatric input. A minority of patients do need extra support and the pretest assessment is helpful in engaging them appropriately (Blochet al, 1992). Most candidates learning of an increased risk should expect to experience some symptoms of depression and anxiety in the first few months as they deal with the immediate impact and shock of the results, but, over a year later, most will experience less depression and live with a heightened perception of the present. A tendency by some to focus on physical symptoms and request frequent neurological examinations has been noted, and it is possible that this could serve to increase anxiety in vulnerable individuals by reinforcing a preoccupation with the illness. Indicators of major psychological problems posttest include significant anxiety or depression pretest, alcohol or other substance misuse, major difficulties coping with previous major life stresses, relationship problems, excessive use of denial, and firm conviction of a decreased risk result.

A surprise finding has been that $10 \%$ have an adverse psychological reaction to a decreased risk result (Huggins et al, 1992). Particularly vulnerable are those who strongly believe that they will receive an increased risk result, and have planned their lives with the firm expectation of dementing and dying prematurely; or those who have unrealistic expectations that it will put everything right about their otherwise difficult and unhappy lives. The first reaction to a 'good' result is intense joy and huge relief. Uncomfortable feelings start to emerge later, for example bottled-up feelings released by the test; grief for lost time spent worrying, past opportunities missed, a happy youth denied; guilt and shock that one is spared while a sibling is not, perhaps heightened by the contradiction of the family's predictions about who would be affected and who not ('the contradiction of preselection'), and difficulties with a change of role as one can no longer be thought of as a potential invalid, or the partner as a potential carer.

It should be remembered that staff involved in presymptomatic counselling may have to watch a destructive untreatable dementing process in 
persons that they have got to know well. The delivery of the news of a definite test result for a fatal dementing illness is an intense emotional experience and debriefing should be arranged as a routine. Moreover, multidisciplinary clinical genetics teams are not used to dealing with issues around suicidal risk in the way that psychiatrists are, and so the psychiatrist's involvement with the presymptomatic counselling process inevitably extends to giving ongoing advice and backup to clinical genetics staff. This works best when organised as a psychiatric liaison service.

\section{Diagnostic testing}

Patients should only be given the test according to their own wishes with fully informed consent and as part of the accepted presymptomatic testing protocol. The test will also have implications for other family members and these need to be considered. Psychiatrists who suspect a patient's mental symptoms may be due to HD should discuss the patient with their local clinical geneticist and not simply take a blood sample and send it to the laboratory. In some areas, there are psychiatrists working in liaison with the clinical genetics service who may assess the patient and advise.

\section{Fragile $X$ syndrome and $X-$ linked mental retardation}

Fragile $X$ syndrome (FRAXA) is an X-linked mental retardation syndrome that was first described as a phenotype by Martin \& Bell in 1943. It is the commonest form of mental retardation after Down's syndrome, occurring with a frequency of 1 in 1250 males and 1 in 2000 females (De Vries et $a l, 1994)$. The classic phenotype is characterised by a triad of mental retardation in the IQ range 35-50 (Glass, 1991), characteristic facies (elongated with facial oedema, tissue thickening, prognathism and large everted ears), and macro-orchidism in adult males. This triad is only present in $60 \%$ of adult fragile $X$ males; $10 \%$ have normal facial features, up to $30 \%$ have no macro-orchidism, and $10 \%$ present with mental retardation alone. A more detailed understanding of the cognitive and behavioural phenotype of FRAXA is beginning to emerge (see Box 2) (Turk, 1992).

\section{An unusual inheritance pattern}

Although it was understood that FRAXA was an $X$-linked disorder, there were some peculiarities about the inheritance which did not conform to classical X-linkage. Males may carry the fragile $X$ mutation and yet be of normal intelligence and appearance, and their chromosomes do not exhibit the fragile site. These males transmit the fragile $X$ chromosome to their daughters who are also phenotypically and cytogenetically normal, but are at risk of having mentally retarded sons. Also, a proportion of female carriers have mild mental retardation, and those that are mentally retarded are more likely to have mentally retarded offspring than are intellectually normal female carriers. Collectively these peculiarities are known as the Sherman paradox.

\section{Cytogenetic testing for FRAXA}

The Martin Bell syndrome was shown to be associated with a cytogenetic abnormality close to the tip of the long arm of the $X$ chromosome (Xq27.3) by Lubs in 1969. The cytogenetic analysis was never wholly reliable in detecting the fragile site, which would not be seen in every meiosis (Rousseau et al, 1991). Patients who tested negative with the old cytogenetic fragile $X$ test should be offered direct mutation analysis. The cytogenetic test is still useful, as it allows other chromosomal abnormalities to be detected.

\section{The molecular pathology of FRAXA}

Until the nature of the FRAXA mutation was elucidated, it was not clear how the observed segregation pattern might arise. The mutation in FRAXA was described in 1991 (Verkerk et al, 1991) and, like the HD mutation, comprises an expanded trinucleotide repeat (CGG) within a gene which has been termed FMR-1. It is normal to have up to 50 CGG repeats in the FMR-1 gene; repeats in the range 50-150 do not interfere with the expression of the FMR-1 gene but are unstable and represent a premutation which expands in length considerably when passing through meiosis in the female. Repeats larger than 150 interfere with the expression of the FMR-1 gene. This explains the Sherman paradox: normal transmitting males have a premutation which is passed to their daughters but remains a premutation. In the formation of germ cells in the daughter's ovary, the premutation is replicated unstably and expands to the full mutation, giving rise to affected individuals in the next generation. Males have one $X$ chromosome and so will always express the abnormal phenotype when they have a full mutation; females with the full mutation may also express the abnormal phenotype, but will not be as badly affected as males because all females are mosaic for the $X$ chromosome and some of their cells will have an active normal $\mathrm{X}$ chromosome. Carrier females with 
Box 2. Fragile $X$ Syndrome: clinical features and behavioural phenotype

Characteristic facies in $\mathbf{9 0 \%}$ of males: long face, large forehead, large simple ears

Macro-orchidism in $70 \%$ of adult males

Joint laxity

Aortic dilatation with mitral valve prolapse

Intellectual impairment is very variable, usually mild to moderate: $I Q$ in the range 35-50

Verbal intelligence exceeds performance abilities in both affected males and normal IQ female carriers

Intellectual development slows with age, particularly after puberty

Speech may be dysfluent with incomplete sentences, echolalia, palilalia, and verbal perseveration, manneristic with compulsive utterances and litanic-like swings of pitch, or cluttered with fast and fluctuating rate, repetitions, poor topic maintenance and tangential comments

Social anxiety with gaze aversion in an otherwise socially responsive individual

Attention and concentration difficulties may be disproportionate to the degree of learning difficulty leading to overactivity

the premutation are of normal phenotype as are males with the premutation.

Direct mutation analysis detects the expansion mutation in the FMR-1 gene with more than a 99\% degree of certainty. This mutation so far accounts for all but a few cases where deletions and point mutations affecting the FMR-1 gene have been described.

\section{FRAXE}

Since it became possible to perform direct mutation analysis in FRAXA, other cytogenetically detectable folate-sensitive fragile sites have now been identified close to the FRAXA site (FRAXE and FRAXF), but slightly more distal (closer to the end of the long arm of the $X$ chromosome). In one of these, FRAXE, mental retardation is also a feature (Knight et al, 1993), but patients do not have the other characteristics of FRAXA. FRAXE is therefore another X-linked mental retardation (XLMR) syndrome caused by a different expanding trinucleotide repeat affecting another gene.

\section{X-linked mental retardation (XLMR)}

Mental retardation (MR) in males is more common than in females partly because of the high prevalence of fragile $X$ syndrome, but also because a number of genes on the $X$ chromosome give rise to MR in the hemizygous male who is not protected from the effects of recessive mutations on the $X$ chromosome. XLMR disorders other than fragile $X$ comprise about 95 separately described syndromes (Schwartz, 1993) which are individually rare, but collectively occur in 1 per 1000 live male births. Some mutations for these disorders have already been found, notably the gene for the neuronal migration disorder $\mathrm{X}$-linked hydrocephalus/MASA syndrome (Jouet et al, 1994), and the gene for Brunner syndrome, a mild mental retardation in physically normal males without dysmorphic features and associated with impulsive aggressive behaviours including rape and arson (Brunner et al, 1993). The former disorder is caused by dysfunction of the neuronal cell adhesion molecule L1CAM which guides neuronal migration during foetal brain development; Brunner's syndrome is caused by deletion of the gene for monoamine oxidase $A$ and such patients have abnormal catecholamine metabolism. Genetic testing is possible in those families where the mutations have been detected, but at present these tests are confined to research laboratories.

\section{Genetic testing and counselling in FRAXA and XLMR}

It is important to recognise FRAXA and XLMR and refer families for genetic testing and counselling as carrier females may be unaware of the risks to their offspring. Testing in individuals with learning disabilities has been shown to be acceptable to their families in centres in the UK and Australia (Young, 1993). In general, X-linked patterns of inheritance are characterised by the presence of affected males in either two generations of a family or in two sibships maternally related. Male to male transmission is absent as a father cannot transmit an X chromosome to a son. The presence of affected females in a family should not exclude the possibility of $X$-linkage as some disorders show heterozygote expression (as does fragile $X$ when the female has the full mutation). Since XLMR comprises a high proportion of MR in males, clinicians should consider XLMR in any male with unexplained MR, particularly when there is any other affected male relative other than the father. It must also be borne in mind that a proportion of male MR without a positive family history will still be due to $X$-linked genes.

The identification of female carriers of $X$-linked 
recessive disorders provides one of the most important opportunities for genetic counselling. In an X-linked recessive disorder, a sister of an affected male will have a prior genetic risk of being a carrier of $1 / 2$, and the niece or first cousin of an affected male will have a prior risk of $1 / 4$. Where the individual female concerned has already had one or more sons, the prior risks are modified. The birth of an affected child confirms carrier status, but, if one or more unaffected sons have been born, the probability of being a carrier is reduced, and this can be calculated using Bayesian analysis (see Harper (1988) for a worked example).

Families where the possibility of FRAXA or other XLMR has been raised need to be referred to a clinical genetics service for an expert dysmorphology opinion. The aim of this will be to examine the family members for features characteristic of described syndromes and to note previously undescribed features. The opinion is also helpful as a prelude to mutation analysis and/or cytogenetic testing where a syndromal diagnosis may suggest that the test should focus down on a particular subchromosomal region. New methods in cytogenetics such as fluorescent in situ hybridisation (FISH) allow much higher resolution analysis than was possible with the earlier staining techniques and are only possible with foreknowledge of a region under suspicion. The referral to a genetics service will also initiate genetic counselling for those family members who wish to be informed of the risks that they are carriers and that their future children could be affected.

\section{Familial Alzheimer's disease}

\section{Early onset Familial Alzheimer's disease (FAD)}

Less than $1 \%$ of Alzheimer's disease is currently recognised as familial with an early age of onset and a Mendelian autosomal dominant pattern of inheritance. Two different genetic loci have been identified to account for these forms of FAD: one on chromosome 21 due to mutations in the amyloid precursor protein (APP) (Goate et al, 1991) and a second locus on the long arm of chromosome 14, where the responsible gene has not yet been found (Clark \& Goate, 1993). Between 15\% and 20\% of FAD is caused by mutations in the APP gene with the greater proportion caused by the gene on chromosome 14.

Predictive testing is possible in families where the mutation in the APP gene is known: patients should be entered into the same counselling protocol as for Huntington's disease and the laboratory testing would be in collaboration with the research group involved in the mutation analysis for that particular family.

\section{Late onset Alzheimer's disease (AD)}

The majority of $\mathrm{AD}$ is of late onset and does not show a simple Mendelian pattern of inheritance, although $40 \%$ of patients with a family history in a first degree relative will show signs of the disease if they survive to the age of 85-90 years (McGuffin et al, 1994). An important advance was made in 1993 when several groups found a robust association between $\mathrm{AD}$ and a particular allele of the gene coding for apolipoprotein E. Apolipoprotein $\mathrm{E}$ (apoE) is a lipoprotein associated with the mobilisation of cholesterol and has been found within the amyloid plaques associated with $\mathrm{AD}$. The apoE gene maps to chromosome $19 q$ where linkage has been demonstrated in some AD families; the apoE gene has three common alleles: $\varepsilon 2$, $\varepsilon 3$ and $\varepsilon 4$. Individuals who are homozygous $\varepsilon 4$ $(\varepsilon 4 / \varepsilon 4)$ are at increased risk of developing $A D$ compared with heterozygotes who have the $\varepsilon 4$ allele in combination with either of the other alleles $(\varepsilon 4 / \varepsilon 2, \varepsilon 4 / \varepsilon 3)$, while $\varepsilon 2$ homozygotes $(\varepsilon 2 / \varepsilon 2)$ are at the lowest risk. The risk is also age related, so that, in multiply affected families, $90 \%$ of homozygotes $(\varepsilon 4 / \varepsilon 4)$ will manifest signs of $A D$ by the age of 80 years (Corder et al, 1993). For those without a family history of $A D$, it has been calculated that homozygotes $(\varepsilon 4 / \varepsilon 4)$ are at $43 \%$ risk of developing the disease by age 85 . Forty per cent of people with $\mathrm{AD}$ do not possess the $\varepsilon 4$ allele, so other aetiological factors must play a part. The allele may also be a risk factor for multi-infarct dementia by conferring an increased risk of atherosclerosis. Testing is unhelpful in differential diagnosis of late onset dementia, and not in use in genetic counselling as its predictive value is too unclear at present (Owen et al, 1994).

\section{References}

Bloch, M., Adam, S., Wiggins, S., et al (1992) Predictive testing for Huntington's disease in Canada: the experience of those receiving an increased risk. American Journal of Medical Genetics, 42, 499-507.

Brunner, H. G., Nelen, M., Breakefield, X. O., et al (1993) Abnormal behaviour associated with a point mutation in the structural gene for monoamine oxidase A. Science, 262, 578-580.

Caine, E. D., \& Shoulson, I. (1983) Psychiatric syndromes in Huntington's disease. American Joumal of Psychiatry, 140, 728733.

Clark, R. F., \& Goate, A. M. (1993) Molecular genetics of Alzheimer's disease. Archives of Neurology, 50, 1164-1172.

Corder, E. H., Saunders, A. M., Strittmatter, W. J., et al (1993) Gene dose of apolipoprotein E type 4 allele and the risk of Alzheimer's disease in late onset families. Science, 261, 921-923.

Craufurd, D. \& Tyler, A. on behalf of the UK Huntington's Prediction Consortium (1992) Predictive testing for Hunt- 
ington's disease: protocol of the UK Huntington's Prediction Consortium. Journal of Medical Genetics, 29, 915-918.

De Vries, L. B. A., Halley, D. J. J., Oostra, B. A., et al (1994) The fragile- $X$ syndrome: a growing gene causing familial intellectual disability. Journal of Intellectual Disability Research, 38, 1-8.

Diamond, R., White, R. F., Myers, R. H., et al (1992) Evidence of presymptomatic cognitive decline in Huntington's disease. Journal of Clinical and Experimental Neuropsychology, 14, 961975.

Duyao, M., Ambrose, C., Myers, R., et al (1993) Trinucleotide repeat length instability and age of onset in Huntington's disease. Nature Genetics, 4, 387-392.

Folstein, S. E., Abbott, M. H., Chase, G. A., et al (1983) The association of affective disorder with Huntington's disease in a case series and in families. Psychological Medicine, 13, 537-542.

- Leigh, J., Parhad, I. M., et al (1986). The diagnosis of Huntington's disease. Neurology, 36, 1279-1283.

Ford, M. F. (1986) Treatment of depression in Huntington's disease with monoamine oxidase inhibitors. British Journal of Psychiatry, 149, 654-656.

Glass, I. A. (1991) X-linked mental retardation. Journal of Medical Genetics, 28, 361-71.

Goate, A., Chartier-Harlin, M. C., Mullan, M., et al (1991) Segregation of a missense mutation in the amyloid precursor protein gene with familial Alzheimer's disease. Nature, 349, 704-706.

Gusella, J. F., Wexler, N. S., Conneally, P. M., et al (1983) A polymorphic DNA marker genetically linked to Huntington's disease. Nature, 306, 234-238.

Harper, P. S. (1988) Practical Genetic Counselling (3rd edn). Butterworth-Heinemann.

Hayden, M. R. (1981) Huntington's Chorea. New York: SpringerVerlag.

Huggins, M., Bloch, M., Wiggins, S., et al (1992) Predictive testing for Huntington's disease in Canada: adverse effects and unexpected results in those receiving a decreased risk. American Journal of Medical Genetics, 42, 508-515.

Huntington's Disease Collaborative Research Group (1993) A novel gene containing a trinucleotide repeat that is expanded and unstable on Huntington's disease chromosomes. Cell, 72, 971-983.

Jouet, M., Rosenthal, A., Armstrong, G., et al (1994) X-linked spastic paraplegia (SPG1), MASA syndrome and X-linked hydrocephalus result from mutations in the $\mathrm{L} 1$ gene. Nature Genetics, 7, 402-407.

Knight, S. J. L., Flannery, A. V., Hirst, M. C., et al (1993) Trinucleotide repeat amplification and hypermethylation of a CpG Island in FRAXE mental retardation. Cell, 74, 127-134.

McGuffin, P., Owen, M., O'Donovan, M., et al (1994) Seminars in Psychiatric Genetics. London: Gaskell.

Owen, M., Liddell, M., \& McGuffin, P. (1994) Alzheimer's disease. An association with apolipoprotein E4 may help unlock the puzzle. British Medical Journal, 308, 672-673.

Pflanz, S., Besson, J.A. O., Ebmeier, K. P., et al (1991) The clinical manifestation of mental disorder in Huntington's disease: a retrospective case record study of disease progression. Acta Psychiatrica Scandanavica, 83, 53-60.

Rousseau, F., Heitz, D., Biancalana, V., et al (1991) Direct diagnosis by DNA analysis of the fragile $X$ syndrome of mental retardation. New England Journal of Medicine, 325,1673-1681.

Schoenfeld, M., Myers, R. H., Cupples, A., et al (1984) Increased rate of suicide among patients with Huntington's disease. Journal of Neurology, Neurosurgery and Psychiatry, 47, 1283-1287.

Schwartz, C. E. (1993) Invited editorial: X-linked mental retardation: in pursuit of a gene map. American Journal of Human Genetics, 52, 1025-1031.

Turk, J. (1992) The fragile X syndrome: on the way to abehavioural phenotype. British Journal of Psychiatry, 160, 24-35.

Tyler, A., Morris, M., Lazarou, L., et al (1992) Presymptomatic testing for Huntington's disease in Wales 1987-90. British Journal of Psychiatry, 161, 481-488.

Verkerk, A. J. M. H., Pieretti, M., Sutcliffe, J. S., et al (1991) Identification of a gene (FMR-1) containing a CGG repeat coincident with a breakpoint cluster region length variation in fragile $X$ syndrome. Cell, 65, 905-914.

Young, I. D. (1993) Diagnosing fragile $X$ syndrome. Lancet, 342, 1004-1005.
The address of the UK Huntington's Disease Association is 108 Battersea High Street, London SW11 $3 H P$.

\section{Multiple Choice Questions}

1 In Huntington's disease:

a bradykinesia is rarely a feature

b first degree relatives of affected individuals are at $50 \%$ risk

c age of onset may be earlier when inheritance is from the mother

d mood disorders are rare

e females are carriers

2 In fragile $X$ syndrome:

a females are never affected, only carriers

b phenotypically normal males may transmit the disorder

c females with the premutation are phenotypically normal

d female sibs of an affected male are always carriers

e female cousins of an affected male might be carriers

3 X-linked mental retardation:

$a$ is genetically heterogenous

b is excluded by direct male to male transmission

c is excluded by occurrence of an affected female in the pedigree

d is always associated with dysmorphic features

e is sometimes sporadic

4 Familial Alzheimer's disease:

a is known to account for a high proportion of Alzheimer's disease

b is autosomal dominant

c is most commonly due to a mutation in the amyloid precursor protein gene

d can be diagnosed presymptomatically with apolipoprotein $\mathrm{E}$ gene testing

e is unlikely to have presenile onset

\begin{tabular}{llll}
\multicolumn{2}{l}{ MCQ answers } \\
1 & 2 & 3 & 4 \\
a F & a F & a T & a F \\
b T & b T & b T & b T \\
c F & c T & c F & c F \\
d F & d F & d F & d F \\
e F & e T & e T & e F
\end{tabular}

